\title{
THE GELFAND-KIRILLOV DIMENSIONS OF ALGEBRAS ARISING FROM REPRESENTATION THEORY
}

\author{
ZONGZHU LIN
}

\begin{abstract}
This note is to study a variety of graded algebras that arise from the induced representations for semisimple algebraic groups and quantum groups. These algebras will play an important role in a study of the cohomology groups of line bundles over the flag varieties. This short note concentrates on the calculation of the Gelfand-Kirillov dimensions of these algebras.
\end{abstract}

\section{INTRODUCTION}

In this paper, we calculate the Gelfand-Kirillov dimensions of certain graded algebras arising from the representation theory of semisimple algebraic groups and quantum groups.

For a given semisimple algebraic group $G$ and a Borel subgroup $B$ over a field $k$. Each rational $B$-module $M$ defines a vector bundle over the flag variety $G / B$ and the space of global sections of the vector bundle is a subspace of $k[G] \otimes M$. In particular $M$ is 1-dimensional, the space of global sections can be viewed as a subspace of the coordinate algebra $k[G]$. If $M$ is a commutative algebra on which $B$ acts as algebra automorphisms, then the space of global sections is also an algebra on which the algebraic group $G$ acts as algebra automorphism. The aim of the paper is to study the properties of these algebra as far as the GK-dimension is concerned. For algebraic groups, the geometry of these algebras can be described. But for their quantum parts, the geometric interpretation still needs to be worked out. So far the evidence shows that in the quantum case, the Gk-dimension is closely related to the space classifying the flag modules of these algebras as studied by Ohn.

For a given line bundle $\mathcal{L}_{\lambda}$ associated to an integral weight $\lambda$ of the root system $R . \quad A(\lambda)=\oplus_{n=0}^{\infty} H^{0}\left(G / B, \mathcal{L}_{\lambda}^{\otimes n}\right)$ is naturally a graded associative algebra with multiplication being the tensor product map $H^{0}\left(G / B, \mathcal{L}_{\lambda}^{\otimes n}\right) \otimes H^{0}\left(G / B, \mathcal{L}_{\lambda}^{\otimes m}\right) \rightarrow$ $H^{0}\left(G / B, \mathcal{L}_{\lambda}^{\otimes(n+m)}\right) . A(\lambda)$ is a rational $G$-module on which $G$ acts as automorphism of graded algebra. If $\lambda$ is not dominant, the algebra $A(\lambda)$ is just the field $k$. For dominant $\lambda$, the algebra $A(\lambda)$ is finitely generated (using the fact that tensor product of two induced modules have a good filtration). The algebra $A(\lambda)$ is actually a subalgebra of the coordinate algebra and thus defines an irreducible projective variety of dimension

1991 Mathematics Subject Classification. Primary 17B55, 20G; Secondary 17B50.

Research was supported in part by NSF grant DMS-9401389. 
GK-dim $(A(\lambda))-1$. This variety actually is the $G$-orbit of the highest weight space in the projective space $\mathbb{P}(V(\lambda))$ of the Weyl module $V(\lambda)$ of highest weight $\lambda$. The geometric property of this algebra is of special interests, in particular, in classifying the point modules of the graded algebra. For any submonoid $\Lambda$ of the dominant weight monoid $X_{+}, M(\Lambda)=\oplus_{\lambda \in \Lambda} k_{\lambda}$ is a $B$-algebra and $H^{0}(G / B, \mathcal{L}(M(\Lambda)))$ is a $G$-algebra and its GK-dimension will also be computed. However, their geometric interpretation is to be worked out.

One can also construct analogous graded algebras $A_{q}(\lambda)$ for quantum groups. Although the algebras $A_{q}(\lambda)$ have the same GK-dimensions as their counter parts for $G$, they fail to be commutative. In this case an interesting question is to classify the point modules and to relate the point modules with the $G$-orbit of the highest weight space in the projective space of the corresponding Weyl modules for the corresponding algebraic group $G$. When, the parameter is not a root of 1 in the field of complex numbers, its known that the quantum coordinate algebra possesses infinite dimensional irreducible modules. But it is not clear whether these subalgebras possesses infinite dimensional irreducible modules. The computation of the GK-dimensions of these algebras works for all characteristic and any non-zero parameter $q$.

This paper is organized as follows. In Section 2, we set more general induction functor for general Hopf algebra (even bialgebras) and prove that the induced module for a Hopf algebra from a Hopf module algebra for a Hopf subalgebra, remains a Hopf module algebra. As a special example, a skew polynomial extension can be viewed as an induced modules over polynomial algebras. Zhang [Zh2] proved skew polynomial extension will increase the GK-dimension by at most 1 . The results in later sections seem to suggest a more general phenomena. This setting of induced representation will cover both algebraic group and quantum group situations. Section 3 is devoted to computing the GK-dimensions of the induced algebras for both quantum groups and algebraic groups. This dimension seems to appear naturally from the geometry of generalized flag varieties. Section 4 contains a few general remarks on interesting appearance of GK-dimensions. In particular the GK-dimension of the extension algebra of a module for a finite dimensional algebra is closely related to the complexity of the modules. For finite groups, it is proved by Carlson $[\mathrm{C}]$ that the Krull dimension (which equals the GK-dimension in this case) of the extension algebra of a finite dimensional module is the same as the complexity of the module. For cocommutative Hopf algebras, the results of Friedlander and Suslin [FS] can be used to prove that the same to be true for all finite dimensional modules.

\section{INDUCED REPRESENTATIONS}

2.1 Let $k$ be a field and $H$ a Hopf algebra over $k$. A full subcategory $\mathcal{C}_{H}$ of the $H$-module category is called admissible if all modules in $\mathcal{C}$ are locally finite, $\mathcal{C}$ includes the trivial modules and is closed under direct limit, tensor product, and finite 
dimensional dual. As studied in [Lin1, Lin2], such category is uniquely determined by a class of cofinite ideals $\mathcal{I}=\left\{\operatorname{Ann}_{H}(M) \mid M \in \mathrm{Ob}\left(\mathcal{C}_{H}\right)\right.$ and $\left.\operatorname{dim} M<\infty\right\}$. For any Hopf subalgebra $D$ of $H$, the collection of ideals $\mathcal{I}_{D}=\{D \cap I \mid I \in \mathcal{I}\}$ defines an admissible category $\mathcal{C}_{D}$ for the Hopf algebra $D$ and the restriction functor from the category of all $H$-modules to the category of all $D$-modules sends all modules in $\mathcal{C}_{H}$ to $\mathcal{C}_{D}$. The restriction functor $\operatorname{Res}_{D}^{H}: \mathcal{C}_{H} \rightarrow \mathcal{C}_{D}$ has a right adjoint functor $\operatorname{Ind}_{D}^{H}: \mathcal{C}_{D} \rightarrow \mathcal{C}_{H}$ which is called the induction functor (see [Lin1, Lin2]).

For any $D$-module $M, \operatorname{Hom}_{D}(H, M)$ is an $H$-module with $H$-action defined by $(h f)(x)=f(x h)$ for all $x, h \in M$ and $f \in \operatorname{Hom}_{D}(H, M)$. If $M$ is in $\mathcal{C}_{D}$, then $\operatorname{Ind}_{D}^{H}(M)$ is the unique largest $H$-submodule of $\operatorname{Hom}_{D}(H, M)$ which is in $\mathcal{C}_{H}$.

Example 1. Let $H=\operatorname{Dist}(G)$ be the distribution algebra of an algebraic group scheme $G$ and $\mathcal{C}_{H}$ be the category of all $G$-rational modules (possibly infinite dimensional). For any closed subgroup scheme $N$ of $G$, Dist $(N)$ is a Hopf subalgebra of $H$ and $\mathcal{C}_{D}$ is exactly the category of all rational $N$-modules and $\operatorname{Ind}_{D}^{H}$ is isomorphic to the induction functor $\operatorname{Ind}_{N}^{G}$ as defined in [Jan] for algebraic group schemes.

Example 2. Let $H=U_{q}(\mathfrak{g})$ be the quantum enveloping algebra, as defined in [L3], of a complex semisimple Lie algebra $\mathfrak{g}$ over $\mathbb{C}(q)$ with $q$ either an indeterminate or $0 \neq q \in \mathbb{C}$. Let $\mathcal{C}_{H}$ be the category of all integrable modules of type 1 . If $D$ is any parabolic subalgebra $U(I, J)$ as defined in $[\mathrm{APW}]$, then the category $\mathcal{C}_{D}$ defined here and the category defined in $[\mathrm{APW}]$ for $U(I, J)$ are the same and the induction functor $\operatorname{Ind}_{D}^{H}$ is also the same as the one defined in $[\mathrm{APW}, 2.1]$.

Proposition 2.2. Let $M$ be a k-algebra. If $M$ is further a D-module algebra [Sw] such that $M$ is in $\mathcal{C}_{D}$ as $D$-module, then $\operatorname{Ind}_{D}^{H} M$ is an $H$-module algebra.

Proof. In deed, since the multiplication map $M \otimes M \rightarrow M$ is a homomorphism of $D$-modules, we have the induced homomorphism $\operatorname{Ind}_{D}^{H} M \otimes \operatorname{Ind}_{D}^{H} M \rightarrow M \otimes M \rightarrow M$ of $D$-modules, which is the composition of the multiplication map with the evaluation map $\operatorname{Ind}_{D}^{H} M \rightarrow M$ [Lin1]. Applying the Frobenius reciprocity [Lin1], we get a homomorphism $\operatorname{Ind}_{D}^{H} M \otimes \operatorname{Ind}_{D}^{H} M \rightarrow \operatorname{Ind}_{D}^{H} M$. One can verify, using the associativity of the multiplication of $M$, that the above defined $H$-module homomorphism is an associative multiplication on $\operatorname{Ind}_{D}^{H} M$. The identity of the multiplication is the counit $\varepsilon: H \rightarrow k 1 \subseteq M$. (By the definition of $D$-module algebra, $k 1$ is a trivial $D$-submodule of $M$.)

Note that $\operatorname{Ind}_{D}^{H}(M)$ is a subalgebra of $\operatorname{Hom}_{D}(H, M)$ which is also a subalgebra of $\operatorname{Hom}_{k}(H, M)$ with the convolution multiplication defined by

$$
(f g)(x)=\sum_{(x)} f\left(x_{(1)}\right) g\left(x_{(2)}\right)
$$

for all $f, g \in \operatorname{Hom}_{k}(H, M)$ and $x \in H$ using Sweedler's notation for comultiplication [Sw]. In particular, when $M=k$ and $D=k, k[H]=\operatorname{Ind}_{k}^{H} k$ is an $H$-module algebra 
(as a subalgebra of the algebra $\operatorname{Hom}_{k}(H, k)$ ). If all modules in $\mathcal{C}_{H}$ are locally finite dimensional, then $k[H]$ has a Hopf algebra structure, which we will not use in this paper. (see [Lin1] for more details.)

Corollary 2.3. If $M=\oplus_{\lambda \in \Lambda} k_{\lambda}$ as D-module algebra with $k_{\lambda}$ being 1-dimensional $D$-module and algebra structure is given by $k_{\lambda} \otimes k_{\mu} \rightarrow k_{\lambda \mu}$ for a set $\Lambda$ of characters of $D$ including $\epsilon$, where $(\lambda \mu)(d)=\lambda\left(d_{(1)}\right) \mu\left(d_{(2)}\right)$ for all $d \in D$, then $\operatorname{Ind}_{D}^{H}(M)=$ $\oplus_{\lambda \in \Lambda} \operatorname{Ind}_{D}^{H} k_{\lambda}$ is isomorphic to an $H$-invariant subalgebra of $k[H]$.

Proof. Using the $H$-module algebra structure of $k[H]$, there is another way to see the $H$-module algebra structure on $\operatorname{Ind}_{D}^{H} M$ as follows. First we can embed $M \otimes_{k} k[H]$ in $\operatorname{Hom}_{k}(H, M)$ by

$$
(a \otimes f)(x)=f(x) a, \quad(\forall x, \in H, f \in k[H], a \in M) .
$$

$k[H]$ has a $(H \otimes H)$-bimodule with actions given by $((h \otimes d) f)(x)=f(\gamma(d) x h)$ for all $x, h, d \in H$ and $f \in k[H]$, where $\gamma: H \rightarrow H$ is the antipode. This makes $k[H]$ an $H \otimes D$-module with $H \otimes D$ being the tensor product Hopf algebra. Note that $k[H]$ is an $H \otimes k=H$-module algebra. By letting $H$ act on $M$ trivially, we can make $M$ an $H \otimes D$-module algebra. But with the above $D \cong k \otimes D$-action, $k[H]$ is not a $D$-module algebra in general. However we have the following

$$
(1 \otimes d)(f g)=\sum_{d}\left(\left(1 \otimes d_{(2)}\right) f\right)\left(\left(1 \otimes d_{(1)}\right) g\right)
$$

For all $d \in D$ and $f, g \in k[H]$. Therefore $k[H] \otimes M$ with the tensor product algebra structure is an $H \otimes D$-module. Note that $D \cong k \otimes D$ is a normal Hopf subalgebra of $H \otimes D$ with quotient Hopf algebra being $H$ [Lin1]. Thus the $D$-fixed point space $(M \otimes k[H])^{D}=\{x \in M \otimes k[H] \mid d(x)=\varepsilon(d) x \forall d \in D\}$ is closed under multiplication and is an $H$-module subalgebra. In fact, for any $f_{i}, g_{j} \in k[H]$ and $a_{i}, b_{j} \in M$, such that $\sum_{i} f_{i} \otimes a_{i}, \sum_{j} g_{j} \otimes b_{j} \in(M \otimes k[H])^{D}$, then

$$
\begin{aligned}
(1 \otimes d)\left(\sum_{i, j} a_{i} b_{j} \otimes f_{i} g_{j}\right) & =\sum_{(d)} \sum_{i, j} d_{(1)}\left(a_{i} b_{j}\right) \otimes\left(1 \otimes d_{(2)}\right)\left(f_{i} g_{j}\right) \\
& =\sum_{(d)} \sum_{i, j}\left(d_{(1)} a_{i}\right)\left(d_{(2)} b_{j}\right) \otimes\left(d_{(4)} f_{i}\right)\left(d_{(3)} g_{j}\right) .
\end{aligned}
$$

Now since $M$ is a direct sum of the 1 -dimensional $D$-modules, we can assume $a_{i}, b_{j}$ are in the 1-dimensional submodules and $\left(d_{(1)} a_{i}\right)$ and $\left(d_{(2)} b_{j}\right)$ are scalars. Apply this to the above one shows that $(M \otimes k[H])^{D}$ is closed under multiplication.

Note that by [Lin1] $\operatorname{Ind}_{D}^{H} M \cong(M \otimes k[H])^{D}$ as $H$-module. Note that $\operatorname{Ind}_{D}^{H} k_{\lambda}=$ $k_{\lambda} \otimes k[H]^{\lambda}$ where $k[H]^{\lambda^{*}}=\{f \in k[H] \mid f(d x)=\lambda(d) f(x), \forall d \in D\}$. We get a natural isomorphism of vector spaces $\oplus_{l a \in \Lambda} \operatorname{Ind}_{D}^{H} k_{\lambda} \rightarrow \oplus_{\lambda \in \Lambda} k[H]^{\lambda^{*}}$ Note that $k[H]^{\lambda^{*}}=\{f \in$ $k[H] \mid(1 \otimes d) f=\lambda(\gamma(d)) f, \forall d \in D\}$ so the notation makes perfect sense. By the definition of the product $\operatorname{Ind}_{D}^{H} k_{\lambda} \otimes \operatorname{Ind}_{D}^{H} k_{\mu} \rightarrow \operatorname{Ind}_{D}^{H}\left(k_{\lambda \mu}\right)$, it is easy to check that 
under the above isomorphism, multiplication is preserved and the multiplication is an isomorphism of $H$-module algebras.

2.4 For each $D$-module $M$ in $\mathcal{C}_{D}$, let $T(M)$ be the tensor algebra of $M . T(M)$ is a (graded) $D$-module algebra. As $D$-module, $T(M)$ is the direct sum of the submodules $M^{\otimes n}$ for $n=0,1, \ldots$ Thus $\operatorname{Ind}_{D}^{H} T(M)=\oplus_{n=0}^{\infty} \operatorname{Ind}_{D}^{H}\left(M^{\otimes n}\right)\left(\operatorname{Ind}_{D}^{H}\right.$ is additive and commutes with direct limits). Furthermore, if $D$ is cocommutative, then the symmetric algebra $S(M)$ is also a grade $D$-module algebra. Thus we can consider the induced algebras from $T(M)$ or from $S(M)$ if $D$ is commutative. An interesting question is to compare that GK-dimension of $\operatorname{Ind}_{D}^{H}(M)$ with the GK-dimension of $M$ for a $D$-module algebra $M$.

2.5 The definition of admissible categories for bialgebras (without the requirement of antipode) and its subalgebra can also be defined by simply removing the condition about dual modules. Then we can define the induced module for bialgebras in a similar way.

Let $A$ be a $k$-algebra and $\sigma$ an algebra endomorphism of $A$. The polynomial extension of $A$ with respect to $\sigma$ is the algebra $A[x, \sigma]$ generated by $A$ and an indeterminate $x$ subject to the commuting condition $x a=\sigma(a) x$. We can make the polynomial algebra $k[y]$ a bialgebra by setting $\Delta(y)=y \otimes y$ and $\varepsilon(y)=1$. Define a new bialgebra $k[y, x]$ with $\Delta(x)=1 \otimes x+x \otimes y$. Then $k[y]$ is a sub-bialgebra of $k[y, x] . k[y]$ acts on $A$ by letting $y \rightarrow \sigma$. Then the multiplication of $A$ is a homomorphism of $k[y]$-modules. We can define the admissible category $\mathcal{C}$ for $k[x, y]$ to be the full subcategory consisting of all $k[x, y]$-modules on which $x$ acts nilpotently. Now the induced algebra $\operatorname{Ind}_{k[y]}^{k[y, x]} A$ is isomorphic to $A[x, \sigma]$. In [Zh2], Zhang proved that $\mathrm{GK}-\operatorname{dim}(A[x, \sigma]) \leq \mathrm{GK}-\operatorname{dim}(A)$. Let $F_{y}$ be the quotient field of the polynomial ring $k[y]$. Note that the GK-dimension of the $F_{y}$-algebra $k[x, y] \otimes_{k[y]} F_{y}$ has GK-dimension of 1 . The results in the next section suggests that something more general along this line should hold. The result should be closely related certain geometry.

\section{Algebras from Representation theory}

3.1 In this section we set $H$ and $\mathcal{C}_{H}$ as in Examples 1-2. Let us fix a root system $R$ with $\Delta \subseteq R^{+}$being the sets of simple roots and positive roots. In the case of Example 1, we assume that $G$ is a simply connected semisimple algebraic group and $N$ is a split Borel subgroup $B$ with a maximal torus $T$. Thus $D$ is the distribution algebra of the Borel subgroup $B$. For any $D$-module $M$ in $\mathcal{C}_{D}$ (thus a rational $B$ module), $\operatorname{Ind}_{D}^{H} M$ is the vector space of global sections of the vector bundle associated to $M$ over the flag variety $G / B$. 
In the case of Example 2, we fix $D$ to be the subalgebra $U(\emptyset, \Delta)$ of $U_{q}(\mathfrak{g})$ generated by $U^{0}, F_{i}^{(m)}$ 's. (See [APW] for more details on the notations.)

Let $X(T)$ be the character group of $T$, which is isomorphic to the integral weight lattice of the root system $R$ [Hu3]. Then all irreducible $D$-modules (up to isomorphism) in $\mathcal{C}_{D}$ in both cases are in one-to-one correspondence to the set $X(T)$, while the irreducible $H$-modules in $\mathcal{C}_{H}$ are in one-to-one correspondence to the set $X_{+}$of all dominant weights in $X(T)$. There is a character of $U^{0}$ associated to each $\lambda \in X(T)$, which is an algebra homomorphism $\lambda: U^{0} \rightarrow \mathbb{C}(q)$ such that $\lambda\left(K_{i}\right)=q^{d_{i}\langle\lambda, \alpha\rangle}$. For each $H$ or $D$-module $M$ and $\lambda \in X(T)$, we denote by $M_{\lambda}$ the $T$ (or $U^{0}$ )-weight space of weight $\lambda$ in the sense of [APW]. In particular each $\lambda$ defines a character for the algebra $D$. Although the Hopf algebra $D$ is not cocommutative, the convolution product of characters is commutative as they factors through the cocommutative Hopf algebra $U^{0}$. Thus we can also regard $X(T)$ as the character group $D$ in the quantum case and write it additively.

3.2 For $H$ and $D$ as mentioned above, we can see that $k[H]$ has no zero divisors. For $\lambda \in X(T), \operatorname{Ind}_{D}^{H} k_{\lambda}=\left(k[H] \otimes k_{\lambda}\right)^{D}=k[H]^{\lambda^{*}}$ where $k[H]^{\lambda^{*}}=\{f \in k[H] \mid(d f)(x)=$ $f(\gamma(d) x)=\lambda(\gamma(d)) f(x)\}$ is the largest $D$-submodule on which $D$ acts via the character $\lambda^{*}=\lambda \circ \gamma$. . In fact, for any $f \in k[H]$, we have $d(f \otimes 1)=\sum\left(d_{(1)} f \otimes d_{(2)} 1\right)=$ $\sum d_{(1)} \lambda\left(d_{(2)}\right) f \otimes 1$. Thus $f \otimes 1 \in \operatorname{Ind}_{D}^{H} k_{\lambda}$ if and only if $d(f \otimes 1)=\varepsilon(d)(f \otimes 1)$ for all $d \in D$ and

$$
\operatorname{Ind}_{D}^{H} k_{\lambda}=\left(k[H] \otimes k_{\lambda}\right)^{D}=\{f \in k[H] \mid d(f \otimes 1)=\varepsilon(d)(f \otimes 1)\} .
$$

In the case of algebraic groups, the above condition is equivalent to $b(f \otimes 1)=(f \otimes 1)$ for all $b \in B$, i.e., $f \otimes 1=b f \otimes \lambda(b) 1$ and $b f=\lambda\left(b^{-1}\right) f$. In the case of quantum groups, the condition is equivalent to $K_{i}(f \otimes 1)=\lambda\left(K_{i}\right) K_{i} f \otimes 1$ and $0=F_{i}(f \otimes 1)=$ $F_{i} f \otimes K_{i}^{-1} 1+f \otimes F_{i} 1=F_{i} f \otimes \lambda\left(K_{i}^{-1}\right)$, i.e., $K_{i} f=\lambda\left(\gamma\left(K_{i}\right)\right) f$ and $F_{i} f=0$ for all $i$. In the case that $q$ is a root of 1 , there are a few more conditions need to be checked, which we omit here.

Let $\Lambda$ be an additive subset of $X(T)$ containing zero, i.e., $\Lambda$ is a submonoid of $X(T)$. We define a $D$-module $M(\Lambda)=\oplus_{\lambda \in \Lambda} k_{\lambda}$. The tensor product $k_{\lambda} \otimes k_{\mu} \rightarrow k_{\lambda+\mu}$ defines a $k$-algebra structure on $M(\Lambda)$. We will discuss the GK-dimension of the algebra $\operatorname{Ind}_{D}^{H} M(\Lambda)$. Since $\operatorname{Ind}_{D}^{H} k_{\lambda}=0$ unless $\lambda \in X_{+}$, we have $\operatorname{Ind}_{D}^{H} M(\Lambda)=\operatorname{Ind}_{D}^{H} M(\Lambda \cap$ $\left.X_{+}\right)$). Thus we can assume that $\Lambda \subseteq X_{+}$.

Note that, by Corollary 2.3, $\operatorname{Ind}_{D}^{H} M(\Lambda)$ is always a subalgebra of the coordinate algebra $k[H]$.

Theorem 3.3. For $\lambda, \mu \in X_{+}$, the multiplication map $\operatorname{Ind}_{D}^{H} k_{\lambda} \otimes \operatorname{Ind}_{D}^{H} k_{\mu} \rightarrow \operatorname{Ind}_{D}^{H} k_{\lambda+\mu}$ is onto. Thus $\operatorname{Ind}_{D}^{H} M(\Lambda)$ is finitely generated if $\Lambda$ is finitely generated as submonoid of $X_{+}$. In particular the coordinate algebra $k[G / U]=\sum_{\lambda \in X_{+}} \operatorname{Ind}_{B}^{G}\left(k_{\lambda}\right)$ is finitely generated for both the algebraic group and its quantum analog. 
Proof. We will prove the first part of the theorem only. The second part is a consequence of the first part. Let $\lambda, \mu \in X_{+} \cdot \operatorname{Ind}_{D}^{H} k_{\lambda} \otimes \operatorname{Ind}_{D}^{H} k_{\mu}$ has a filtration with consecutive quotients being of the form $\operatorname{Ind}_{D}^{H} k_{\nu}$ with $\nu \in X_{+}$. For semisimple algebraic groups this is well-known [M, Don, W] (for the characteristic 0 situation, this is trivial due to the semi-simplicity of finite dimensional modules and the characterization of the simple modules by Borel and Weil). For quantum groups when the parameter $q$ is not a root of unit, this is similar to the case of characteristic 0 reductive groups with detailed work in [APW]. When $q$ is a $p^{r}$-th root of 1 , Andersen, Polo, and Wen have shown the result in [APW]. The general situation is proved by Paradowski $[\mathrm{P}]$ using Lusztig's canonical basis.

To show that $\operatorname{Ind}_{D}^{H} k_{\lambda} \otimes \operatorname{Ind}_{D}^{H} k_{\mu} \rightarrow \operatorname{Ind}_{D}^{H} k_{\lambda+\mu}$ is onto, one first notes that the dual module of $\operatorname{Ind}_{D}^{H} k_{\lambda}$ is a universal highest weight module, which is called a Weyl module (or the quantum Weyl module). The dual module of $\operatorname{Ind}_{D}^{H} k_{\lambda} \otimes \operatorname{Ind}_{D}^{H} k_{\mu}$ admits a filtration with consecutive quotient being Weyl modules. Now using almost identical argument as in the proof of [W, (3.1)] one can show that the dual module of $\operatorname{Ind}_{D}^{H} k_{\lambda+\mu}$ is a submodule of the dual module of $\operatorname{Ind}_{D}^{H} k_{\lambda} \otimes \operatorname{Ind}_{D}^{H} k_{\mu}$. This shows that $\operatorname{Ind}_{D}^{H} k_{\lambda+\mu}$ is a quotient of $\operatorname{Ind}_{D}^{H} k_{\lambda} \otimes \operatorname{Ind}_{D}^{H} k_{\mu}$. Now we conclude that the multiplication map is onto since $\operatorname{Hom}_{H}\left(\operatorname{Ind}_{D}^{H} k_{\lambda} \otimes \operatorname{Ind}_{D}^{H} k_{\mu}, \operatorname{Ind}_{D}^{H} k_{\lambda+\mu}\right)$ is 1-dimensional.

Note that, when $\Lambda=X(T)_{+}, \operatorname{Ind}_{B}^{G}(M(\Lambda)$ is the shape algebra of the flag variety $G / B$. In the quantum case, $\operatorname{Ind}_{D}^{H}(M(\Lambda))$ is the shape algebra of the quantum flag variety. In particular the graded algebra $\operatorname{Ind}_{D}^{H}(M(\Lambda))$ is generated by the degree 1 space, where the gradation is given by the sum of heights of the highest weights. Ohn [O] was interested in studying the algebra $\operatorname{Ind}_{D}^{H} M(\Lambda)$, especially, its geometric properties.

3.4 Let $f: \mathbb{N} \rightarrow \mathbb{N}$ be a function. The polynomial growth of $f$ is defined to be the real number $\nu(f)=\inf \left\{\nu \in \mathbb{R} \mid f(n) \leq n^{\nu}\right.$ for all $\left.n \gg 0\right\}$. Let $A$ be a $k$ algebra. Suppose $A$ is finitely generated. Let $V \subseteq A$ be a subspace containing a set of generators of $A$. Set $A_{n}=\sum_{i=1}^{n} V^{i} \subseteq A$ with $V^{0}=k 1$ and $f(n)=\operatorname{dim}\left(A_{n}\right)$. The GK-dimension (Gelfand-Kirillov dimension) of $A$ is defined to be $\nu(f)$. The GK-dimension of $A$ is independent of the choice of the subspace $V$ and denoted by GK-dim $(A)$. For more general $k$-algebras $A$, the GK-dimension of $A$ is defined to be $\sup \left\{\mathrm{GK}-\operatorname{dim}\left(A^{\prime}\right) \mid A^{\prime}\right.$ is a finitely generated subalgebra of $\left.A\right\}$. In particular, if $f(n)=\operatorname{dim}\left(A_{n}\right)$ is polynomial of $n$, then GK-dim $(A)=\operatorname{deg}(f(n))$. See $[\mathrm{MR}]$ and $[\mathrm{KL}]$ for more details about GK-dimensions of algebras and modules.

3.5 To calculate the GK-dimension of the algebra $\operatorname{Ind}_{D}^{H} M(\Lambda)$ for any finitely generated submonoid $\Lambda \subseteq X_{+}$. We consider the special case $\Lambda=\{n \lambda \mid n \in \mathbb{N}\}$ for fixed $\lambda \in X_{+}$. The algebra $A=\operatorname{Ind}_{D}^{H} M(\Lambda)$ is naturally an $\mathbb{N}$-graded algebra. Theorem 3.3 shows that the subspace $\operatorname{Ind}_{D}^{H} k_{\lambda}$ generates the algebra $\operatorname{Ind}_{D}^{H} M(\Lambda)$. By Weyl's 
character formula [Hu3] we have

$$
\operatorname{dim} \operatorname{Ind}_{D}^{H} k_{\lambda}=\frac{\prod_{\alpha \in R^{+}}\langle\lambda+\rho, \alpha\rangle}{\prod_{\alpha \in R^{+}}\langle\rho, \alpha\rangle}=\prod_{\alpha \in R^{+}}\left(\frac{\langle\lambda, \alpha\rangle}{\langle\rho, \alpha\rangle}+1\right) .
$$

Here $\rho$ is the half sum of all positive roots, and $\langle\lambda, \alpha\rangle=\frac{2(\lambda, \alpha)}{(\alpha, \alpha)}$ with $(, \quad)$ being the inner product on the Euclidean space containing the root system $R$.

For the convenience of later calculation, we set $\operatorname{dim}(\lambda)=\operatorname{dim} \operatorname{Ind}_{D}^{H} k_{\lambda}$ and take $V=\operatorname{Ind}_{D}^{H} k_{\lambda}$. Theorem 3.3 shows that $V^{n}=\operatorname{Ind}_{D}^{H} k_{n \lambda}$. Thus $\operatorname{dim} V^{n}=\operatorname{dim}(n \lambda)$ for all $n$. By the definition of the GK-dimension, [MR, 8.1.9] we need to calculate the dimension of $A_{n}=\sum_{i=0}^{n} V^{i}$. Note that

$$
\operatorname{dim} A_{n}=\sum_{i=0}^{n} \operatorname{dim} V^{i}=\sum_{i=0}^{n} \operatorname{dim}(i \lambda)=\sum_{i=0}^{n} \prod_{\alpha \in R^{+}}\left(\frac{\langle\lambda, \alpha\rangle}{\langle\rho, \alpha\rangle} i+1\right) .
$$

3.6 For fixed $\lambda, \operatorname{dim}(i \lambda)$ is a polynomial of $i$, i.e., $\operatorname{dim}(i \lambda)=\sum_{s=0}^{N} a_{s} i^{s}$ with $a_{s} \in \mathbb{Q}$ depending on $\lambda$ only. In particular $a_{N}=\prod_{\alpha \in R^{+}} \frac{\langle\lambda, \alpha\rangle}{\langle\rho, \alpha\rangle}$. Here $N=\left|R^{+}\right|$is the number of positive roots.

Define $f(n)=\operatorname{dim}\left(A_{n}\right)$. We regard $f(n)$ as a function of $n$. Then

$$
f(n)=\sum_{i=0}^{n} \sum_{s=0}^{N} a_{s} i^{s}=\sum_{s=0}^{N} a_{s}\left(\sum_{i=0}^{n} i^{s}\right) .
$$

The following lemma is well-known, but we can not locate a reference. Since we are going to use it repeatedly, we give a proof here for the completeness.

Lemma 3.7. For any nonnegative integer $s, \sum_{i=0}^{n} i^{s}=\frac{1}{s+1} n^{s+1}+t_{n}$. Here $t_{n}$ is a polynomial of $n$ of degree at most s.

Proof. When $s=0,1$, this is trivial. We now use induction on $s$. Denote $g_{s}(n)=$ $\sum_{i=1}^{n} i^{s}$. Assume that $g_{s}(n)=a_{s+1} n^{s+1}+a_{s} n^{s}+\cdots a_{0}$ is a polynomial of $n$ with coefficients $a_{0}, a_{1}, \ldots a_{s+1}$ independent of $n$ and $a_{s+1}=\frac{1}{s+1}$. By using the Abel partial summation formula (or integration by parts) we one gets

$$
\begin{aligned}
g_{s+1}(n) & =g_{s}(n)(n+1)-\sum_{i=1}^{n} g_{s}(i) \\
& =(n+1) \sum_{j=0}^{s+1} a_{j} n^{j}-\sum_{j=0}^{s+1} a_{j}\left(\sum_{i=1}^{n} i^{j}\right) \\
& =(n+1) \sum_{j=0}^{s+1} a_{j} n^{j}-\frac{1}{s+1} g_{s+1}(n)-\sum_{j=0}^{s} a_{j} g_{j}(n)
\end{aligned}
$$


Thus, solving out $g_{s+1}(n)$ we get

$$
g_{s+1}(n)=\frac{1}{s+2} n^{s+2}+\text { terms with the degrees of } n \text { at most } s+1 .
$$

Hence the induction proves the lemma.

Note that more generally we have $\nu\left(g_{s}(n)\right)=s+1$ for any $s>0$. In fact we can write $g_{s}(n)=n^{s+1} \cdot \frac{1}{n} \sum_{i=1}^{n}\left(\frac{i}{n}\right)^{s}$ and $g_{s}(n) / n^{s+1} \rightarrow \int_{0}^{1} x^{s} d x=1 /(s+1)$.

Let us go back to (3.3). $f(n)=\sum_{s=0}^{N} a_{s} g_{s}(n)$ is a polynomial of degree $N+1$ by the above lemma if $\langle\lambda, \alpha\rangle>0$ for all simple roots $\alpha$.

For more general weight $\lambda$, we consider the polynomial

$$
d_{\lambda}(x)=\prod_{\alpha \in R^{+}}\left(\frac{\langle\lambda, \alpha\rangle}{\langle\rho, \alpha\rangle} x+1\right) .
$$

Let $R_{\lambda}^{+}$be the set of all positive roots $\alpha$ such that $\langle\lambda, \alpha\rangle=0$. Then $d_{\lambda}(x)$ has degree $N-\left|R_{\lambda}^{+}\right|$. Thus $f(n)$ has degree $N-\left|R_{\lambda}^{+}\right|+1$.

Theorem 3.8. The graded algebra $A(\lambda)=\oplus_{n=0}^{\infty} \operatorname{Ind}_{D}^{H} k_{n \lambda}$ has GK-dimension $N+$ $1-\left|R_{\lambda}^{+}\right|$.

Let $I_{\lambda}=\left\{\alpha \in \Delta \mid\left\langle\lambda, \alpha^{\vee}\right\rangle=0\right\}$ and let $P_{\lambda}$ be the parabolic subgroup containing $B$ and positive simple roots $\alpha \in I_{\lambda}$. Then the one dimensional $B$-modules $k_{n \lambda}$ extends to $P_{\lambda}$-module structures. Thus $\operatorname{Ind}_{B}^{G}\left(k_{n \lambda}\right)=\operatorname{Ind}_{P_{\lambda}}^{G} k_{n \lambda}$, which is the space of global sections of the line bundle $k_{n \lambda}$ on $G / P_{\lambda}$. Note that $\operatorname{dim} G / P_{\lambda}=N-\left|R_{\lambda}^{+}\right|$. The theorem shows that the GK-dimension of the algebra $\operatorname{Ind}_{P_{\lambda}}^{G} \oplus_{n=0}^{\infty} k_{n \lambda}$ is $\operatorname{dim}\left(G / P_{\lambda}\right)+1$. In the quantum version, we don't have a precise geometric definition of generalized flag manifold $G / P_{\lambda}$. However the GK-dimension of the quantum analog of the graded algebra $\operatorname{Ind}_{P_{\lambda}}^{G}\left(\oplus_{n=0}^{\infty} k_{n \lambda}\right.$ is independent of the choice of the weight $\lambda$. It depends on the set $R_{\lambda}^{+}$only. This dimension seams to be the dimension of the algebra variety that parameterizes the isomorphism classes of the point modules of the the graded algebras. Later we will see that variety is independent of the choice of the weight $\lambda$.

3.9 As mentioned in the introduction, the graded algebra $A(\lambda)$ defines an irreducible projective variety $Y_{\lambda}$ in the commutative case. In fact, using the Serre duality, $\operatorname{Ind}_{D}^{H}\left(k_{\lambda}\right)$ is the space of linear functions on the Weyl module $V(\lambda)$. Theorem 3.3 shows that induced algebra homomorphism $k[V(\lambda)] \rightarrow A(\lambda)$ is homogeneous and surjective. Thus we can embed $Y_{\lambda}$ in $\mathbb{P}(V(\lambda))$ as $G$-invariant closed subvariety. In fact, $Y_{\lambda}$ the homogeneous subvariety generated by highest weight space. Since $P_{\lambda}$ stabilizes the highest weight space, then we have a natural morphism $G / P_{\lambda} \rightarrow Y_{\lambda}$. By comparison of the dimension and the fact that both varieties are complete, therefore, the above map is finite. In the quantum case, In the quantum case, it is interesting to classify the point modules of the algebra $A(\lambda)$ and relate the variety of all point modules in the sense of Artin-Tate-Van der Bergh [ATV] to the variety $Y_{\lambda}$ or $G / P_{\lambda}$. 
3.10 Next we consider the case when $\Lambda=\sum_{i=1}^{r} \mathbb{N} \lambda_{i} \subseteq X_{+}$is a free commutative submonoid of rank $r$ with basis $\lambda_{1}, \lambda_{2}, \ldots, \lambda_{r}$. For example $\Lambda=X_{+}$is free commutative monoid of rank $l$ with basis $\omega_{1}, \ldots, \omega_{l}$ being the set of fundamental weights. Here $l$ is the rank of the root system. By Theorem 3.3, then algebra $A=\operatorname{Ind}_{D}^{H} M(\Lambda)$ is generated by the subspace $V=\oplus_{i=1}^{R} \operatorname{Ind}_{D}^{H} k_{\lambda_{i}}$.

$$
A_{n}=\sum_{i=0}^{n} V^{i}=\bigoplus_{\substack{\lambda \in X_{+} \\ \lambda=\sum n_{i} \lambda_{i}, \sum n_{i} \leq n}} \operatorname{Ind}_{D}^{H} k_{\lambda}
$$

Then $\operatorname{dim} A_{n}=\sum \operatorname{dim}\left(\sum_{i=1}^{r} n_{i} \lambda_{i}\right)$ with the summation taken over all $\left(n_{1}, \ldots, n_{r}\right) \in$ $\mathbb{N}^{r}$ such that $\sum n_{i} \leq n$. Using Weyl's dimension formula (3.1) we have

$$
\operatorname{dim}\left(\sum n_{i} \lambda_{i}\right)=\prod_{\alpha \in R^{+}}\left(1+\sum_{i=1}^{r} n_{i} \frac{\left\langle\lambda_{i}, \alpha\right\rangle}{\langle\rho, \alpha\rangle}\right)=\prod_{\alpha \in R^{+}}\left(1+\sum_{i=1}^{r} n_{i} a_{i}(\alpha)\right)
$$

Here $a_{i}(\alpha)=\frac{\left\langle\lambda_{i}, \alpha\right\rangle}{\langle\rho, \alpha\rangle}$ (for $\left.i=1, \cdots, r\right)$ are non-negative rational numbers.

More generally, let us fix an $l \in \mathbb{N}$. For each $n \in \mathbb{N}$, set $C(l, n)=\left\{\left(n_{1}, \ldots, n_{l}\right) \in\right.$ $\left.\mathbb{N}^{l} \mid \sum_{i=1}^{l} n_{i}=n\right\}$. For each $t=\left(t_{1}, \ldots, t_{l}\right) \in \mathbb{N}^{l}$, we define

$$
d_{l, t}(n)=\sum_{\left(n_{1}, \ldots, n_{l}\right) \in C(l, n)} n_{1}^{t_{1}} \cdots n_{l}^{t_{l}}
$$

Note that $d_{l, t}(n)$ generalizes the function $g_{s}(n)$. In fact $g_{s}(n)=d_{2,(s, 0)}(n)$. The following lemma generalizes Lemma 3.7.

Lemma 3.11. For each $t=\left(t_{1}, \ldots t_{l}\right) \in \mathbb{N}^{l}, d_{l, t}(n)$ is a polynomial of $n$ with degree $l-1+t_{1}+\cdots+t_{l}$ and the leading coefficient $\frac{t_{1} ! \cdots t_{l} !}{\left(l-1+t_{1}+\cdots+t_{l}\right) !}$.

Proof. We use induction on $l$. It is trivial when $l=1$. Assume $l>1$. We write

$$
\begin{aligned}
d_{l, t}(n) & =\sum_{n_{1}=0}^{n} n_{1}^{t_{1}}\left(\sum_{\left(n_{2}, \ldots, n_{l}\right) \in C\left(l-1, n-n_{1}\right)} n_{2}^{t_{2}} \cdots n_{l}^{t_{l}}\right) \\
& =\sum_{n_{1}=0}^{n} n_{1}^{t_{1}} d_{l-1, t^{\prime}}\left(n-n_{1}\right)=\sum_{i=0}^{n}(n-i)^{t_{1}} d_{l-1, t^{\prime}}(i) .
\end{aligned}
$$

Here $t^{\prime}=\left(t_{2}, \ldots, t_{l}\right) \in \mathbb{N}^{l-1}$. By the induction assumption, we can write $d_{l-1, t^{\prime}}(i)=$ $\sum_{j=0}^{r} c_{j} i^{j}$ with $r=l-2+t_{2}+\cdots+t_{l}, c_{0}, c_{1}, \ldots, c_{r}$ being independent of $i$, and 
$c_{r}=\frac{t_{2} ! \cdots t_{l} !}{\left(l-2+t_{2}+\cdots+t_{l}\right) !}$. Then, by using Lemma 3.7, we have

$$
\begin{aligned}
\sum_{i=0}^{n}(n-i)^{t_{1}} d_{l-1, t^{\prime}}(i) & =\sum_{i=0}^{n} \sum_{s=0}^{t_{1}}\left(\begin{array}{c}
t_{1} \\
s
\end{array}\right)(-1)^{s} n^{t_{1}-s} i^{s} \sum_{j=0}^{r} c_{j} i^{j} \\
& =\sum_{j=0}^{r} c_{j} \sum_{s=0}^{t_{1}}\left(\begin{array}{c}
t_{1} \\
s
\end{array}\right)(-1)^{s} n^{t_{1}-s} \sum_{i=0}^{n} i^{s+j} \\
& =\sum_{j=0}^{r} c_{j} \sum_{s=0}^{t_{1}}\left(\begin{array}{c}
t_{1} \\
s
\end{array}\right)(-1)^{s} n^{t_{1}-s}\left(\frac{1}{s+j+1} n^{s+j_{1}}+\cdots\right) \\
& =\sum_{j=0}^{r} c_{j}\left[\left(\sum_{s=0}^{t_{1}}\left(\begin{array}{c}
t_{1} \\
s
\end{array}\right)(-1)^{s} \frac{1}{s+j+1}\right) n^{t_{1}+j+1}+\cdots\right] .
\end{aligned}
$$

The leading term occurs when $j=r$ with leading coefficient being

$$
\sum_{s=0}^{t_{1}}\left(\begin{array}{l}
t_{1} \\
s
\end{array}\right) \frac{(-1)^{s}}{s+r+1}
$$

Since $x^{r}(1-x)^{t_{1}}=\sum_{s=0}^{t_{1}}\left(\begin{array}{c}t_{1} \\ s\end{array}\right)(-1)^{s} x^{r+s}$, by the definition of the classical Beta-function, we have

$$
B\left(r+1, t_{1}+1\right)=\int_{0}^{1} x^{r}(1-x)^{t_{1}} d x=\sum_{s=0}^{t_{1}}\left(\begin{array}{c}
t_{1} \\
s
\end{array}\right) \frac{(-1)^{s}}{s+r+1} .
$$

Here $B(\alpha, \beta)$ is the classical beta-function and $B\left(r+1, t_{1}+1\right)=\frac{r ! t_{1} !}{\left(r+t_{1}+1\right) !}>0$ since $r$ and $t_{1}$ are non-negative integers $(l \geq 2)$. Thus the leading coefficient is $c_{r} \frac{r ! t_{1} !}{\left(r+t_{1}+1\right) !}=$ $\frac{t_{2} ! \cdots t_{l} !}{\left(l-2+t_{2}+\cdots+t_{l}\right) !} \frac{r ! t_{1} !}{\left(r+t_{1}+1\right) !}=\frac{t_{1} ! \cdots t_{l} !}{\left(l-1+t_{1}+\cdots+t_{l}\right) !}$.

Proposition 3.12. For positive integers $r, N$, and non-negative real numbers $a_{i j}$, with $i=1, \ldots, r$, set $J=\left\{j \mid \sum_{i=1}^{r} a_{i j}=0\right\}$. Then

$$
d(n)=\sum_{\left(n_{1}, \cdots, n_{r}\right) \in C(r, n)} \prod_{j=1}^{N}\left(1+\sum_{i=1}^{r} n_{i} a_{i j}\right)
$$

is a polynomial of $n$ with degree $N+r-1-|J|$.

Proof. Let us consider the polynomial $f\left(x_{1}, \cdots, x_{r}\right)=\prod_{j=1}^{N}\left(\sum_{i=1}^{r}\left(x_{i} a_{i j}+1\right)\right)$. Then the polynomial $f\left(x_{1}, \cdots, x_{r}\right)$ has total degree $N-|J|$ since each factor has degree 1 unless $j \in J$. Without lose of generality, we can assume that $|J|=0$. Let us write 


$$
\begin{aligned}
f\left(x_{1}, \cdots, x_{r}\right) & =\sum c_{t_{1}, \cdots t_{r}} x_{1}^{t_{1}} \cdots x_{r}^{t_{r}} \text {. Then } \\
d(n) & =\sum_{\left(n_{1}, \cdots, n_{r}\right) \in C(r, n)} f\left(n_{1}, \cdots, n_{r}\right) \\
& =\sum_{\left(t_{1}, \cdots, t_{r}\right)} c_{t_{1}, \cdots, t_{r}}\left(\sum_{\left(n_{1}, \cdots, n_{r}\right) \in C(r, n)} n_{1}^{t_{1}} \cdots n_{r}^{t_{r}}\right)=\sum_{\left(t_{1}, \cdots, t_{r}\right)} c_{t_{1}, \cdots, t_{r}} d_{r, t}(n) .
\end{aligned}
$$

By the lemma above, $d_{r, t}(n)$ is a polynomial of $n$ with degree $t_{1}+\cdots+t_{r}+r-1$ while the total degree of $f\left(x_{1}, \cdots, x_{r}\right)$ is $N$. Note that all $c_{t_{1}, \cdots, t_{r}}$ are sums of products of $a_{i j}$ 's. Thus monomials of highest total degree $N$ in $f\left(x_{1}, \cdots, x_{r}\right)$ must have nonnegative coefficients with at least one being positive. On the other hand, the leading coefficients of all $d_{r, t}(n)$ 's are positive. Therefore the coefficient of $n^{N+r-1}$ (the leading term) in $d(n)$ must be positive. Hence $d(n)$ has degree $N+r-1$ since $N=t_{1}+\cdots+t_{r}$ for any monomial $x_{1}^{t_{1}} \cdots x_{r}^{t_{r}}$ with total degree $N$.

Let us write $d(n)=\sum_{j=0}^{N+r-1} d_{j} n^{j}$ for all $n \in \mathbb{N}$. Here $d_{j}$ 's are independent of $n$ and $d_{N+r-1}>0$.

Theorem 3.13. When $\Lambda \subseteq X_{+}$is a free commutative submonoid of rank $r$ and $I_{\Lambda}=\left\{\alpha \in \Delta \mid\left\langle\lambda, \alpha^{\vee}\right\rangle=0, \forall \lambda \in \Lambda\right\}$, then the GK-dimension of $\operatorname{Ind}_{D}^{H} M(\Lambda)$ is $\operatorname{dim} G / P_{\Lambda}+r$, where $P_{\Lambda}$ is the parabolic subgroup containing $B$ determined the simple roots in $I_{\Lambda}$.

Proof. Let $J_{\Lambda}=\left\{\alpha \in R^{+} \mid\left\langle\lambda, \alpha^{\vee}\right\rangle=0, \forall \lambda \in \Lambda\right\}$. Note that

$$
\begin{aligned}
\operatorname{dim} A_{n} & =\sum_{i=0}^{n} d(i)=\sum_{i=0}^{n}\left(\sum_{j=0}^{N+r-1-|J|} d_{j} i^{j}\right) \\
& =\sum_{j=0}^{N+r-1-|J|} d_{j}\left(\sum_{i=0}^{n} i^{j}\right) \\
& =\sum_{j=0}^{N+r-1-|J|} d_{j}\left(\frac{1}{j+1} n^{j+1}+\cdots\right) .
\end{aligned}
$$

Thus $\operatorname{dim} A_{n}$ is a polynomial of $n$ with the leading term occurs when $j=N+r-1-|J|$ and the leading coefficient being $\frac{d_{N+r-1-|J|}}{N+r-|J|}$. Note that $\operatorname{dim}\left(G / P_{\Lambda}\right)=N-|J|$. The theorem follows.

3.14 A rather more general question is to compute the GK-dimension of $\operatorname{Ind}_{D}^{H}(M(\Lambda))$ for more general submonoid $\Lambda$. Note that all submonoid of $X_{+}$is finitely generated. Thus the algebra $\operatorname{Ind}_{D}^{H}(M(\Lambda))$ needs not be finitely generated. However, let $L=\mathbb{Z} \Lambda$ 
be the $\mathbb{Z}$-submodule of $X(T)$ generated by $\Lambda$. Then $L$ is finitely generated and free. We need the following lemma.

Lemma 3.15. If $L$ is a $\mathbb{Z}$-submodule of $\mathbb{Z}^{n}$ generated by a subset $Y$ consisting of elements of $\mathbb{Z}^{n}$ with non-negative components, then $L$ has basis $\left\{\lambda_{1}, \ldots, \lambda_{r}\right\}$ such that $Y \subseteq \sum_{i=1}^{r} \mathbb{N} \lambda_{i}$. In general, $\lambda_{1}, \ldots, \lambda_{r}$ don't have non-negative components.

Proof. We use induction on $n$. When $n=1$, the lemma is trivial. For $n>1$, let $\left\{e_{1}, \ldots, e_{n}\right\}$ be the standard basis of $\mathbb{Z}^{n}$. We consider the projection $\phi: \mathbb{Z}^{n} \rightarrow \mathbb{Z}^{n-1}$ to the first $n-1$ components. Let $\bar{L}=\phi(L)$ and $\bar{Y}=\phi(Y)$. Then $\bar{L}$ in $\mathbb{Z}^{n-1}$ is generated by $\bar{Y}$. By the induction hypothesis, $\bar{L}$ has a $\mathbb{Z}$-basis $\left\{\bar{\lambda}_{1}, \ldots, \bar{\lambda}_{r^{\prime}}\right\}$ such that $\bar{Y} \subseteq \sum_{i=1}^{r^{\prime}} \mathbb{N} \bar{\lambda}_{i}$. If $\phi: L \rightarrow \bar{L}$ is not an isomorphism, then the kernel is a rank one free $\mathbb{Z}$-submodule with a basis $a e_{n}$ for some positive integer $a$. In this case, $\lambda_{i}=\left(\bar{\lambda}_{i}, 0\right) \in L$ for $i=1, \ldots, r^{\prime}$ and $\left\{\lambda_{1}, \ldots, \lambda_{r^{\prime}}, a e_{n}\right\}$ is the required basis.

If $\phi: L \rightarrow \bar{L}$ is an isomorphism of $\mathbb{Z}$-modules, let $\lambda_{i} \in L$ such that $\phi\left(\lambda_{i}\right)=\bar{\lambda}_{i}$ for $i=1, \ldots, r^{\prime}$. Then $\lambda_{i}=\left(\bar{\lambda}_{i}, a_{i}\right)$ for uniquely determined $a_{i} \in \mathbb{Z}$. Each element of $Y$ is of the form $y=(\bar{y}, a)$ with $\bar{y} \in \bar{Y}$ and $a \in \mathbb{N}$. Then $\bar{y}=\sum_{i=1}^{r^{\prime}} x_{i} \bar{\lambda}_{i}$ for some $x_{i} \in \mathbb{N}$. Then $y=\sum_{i=1}^{r^{\prime}} x_{i} \lambda_{i}$ since $\phi$ is an isomorphism between $L$ and $\bar{L}$.

We remark that in general one can not take the basis with non-negative components, even having $\mathbb{Z}$ replaced by the fields $\mathbb{Q}$ and $\mathbb{R}$ of rational numbers and real numbers respectively. The above inductive argument can prove that, for $n=1,2,3$, if $L$ is a $\mathbb{Q}$-subspace generated by vectors with non-negative components, then $L$ has a basis with non-negative components such that such that all vectors in $L$ with non-negative components are non-negative linear combinations of the basis element. In general when $\operatorname{dim} L=1$ or $n$, the statement is trivial. Thus the situations for $n=1,2$ are trivial. For $n=3$, one only needs to consider $\operatorname{dim} L=2$. If one can take one of the three projections $\phi$ which is not an isomorphism on $L$, then one of the standard basis element is in $L$ and the induction above will finish the proof. The only case the above proof needs more attention is that $\phi$ is an isomorphism and one of $a_{i}$ is positive and the other is negative. Now one can easily replace the one with negative $a_{i}$ by a non-negative linear combination of two $\lambda_{i}$ with zero in the third component.

For $n>3$, one can take $L$ to be the solution space of $x_{1}+x_{2}=x_{3}+x_{4}$ in $\mathbb{R}^{4}$. $L$ is generated by $(1,0,1,0),(1,0,0,1),(0,1,1,0),(0,1,0,1)$. But $L$ does not have a $\mathbb{R}$ basis with non-negative components such that these four generators are non-negative linear combinations of the basis vectors.

3.16 We are now ready to compute the GK-dimension of $\operatorname{Ind}_{D}^{H}(M(\Lambda))$ for any submonoid $\Lambda$ of $X_{+}$. Let $L=\mathbb{Z} \Lambda$ be the free $\mathbb{Z}$-submodule of rank $r$. The subsets $I_{L} \subseteq \Delta$ and $J_{L} \subseteq R^{+}$are defined similarly. Then we have $I_{\Lambda}=I_{L}$ and $J_{\Lambda}=J_{L}$. Let $P_{\Lambda}$ be the parabolic subgroup of $G$ containing $B$ with $I_{\Lambda}$ being the simple roots of the Levi factor. 
Theorem 3.17. For any submonoid $\Lambda \subseteq X_{+}$, the GK-dimension of the algebra $\left.\operatorname{Ind}_{D}^{H} M(\Lambda)\right)=\operatorname{dim}\left(G / P_{\Lambda}\right)+1$.

Proof. First of all $\mathbb{Q} \Lambda$ has a $\mathbb{Q}$-basis $\left\{\lambda_{1}, \ldots, \lambda_{r}\right\} \subseteq \Lambda$. Let $\Lambda^{\prime}=\sum_{i=1}^{r} \mathbb{N} \lambda_{i} \subseteq \Lambda$. Note that every element of $\Lambda$ is a $\mathbb{Q}$-linear combination of $\lambda_{1}, \lambda_{2}, \ldots, \lambda_{r}$. Thus we have $I_{\Lambda}=I_{\Lambda^{\prime}}$ and $J_{\Lambda}=J_{\Lambda^{\prime}}$. Therefore, $P_{\Lambda}=P_{\Lambda^{\prime}}$. Since $\operatorname{Ind}_{D}^{H} M\left(\Lambda^{\prime}\right)$ is a subalgebra of $\operatorname{Ind}_{D}^{H} M(\Lambda)$, by Theorem 3.13, the GK-dimension of $\operatorname{Ind}_{D}^{H} M(\Lambda)$ is at least $\operatorname{dim} G / P+$ 1.

We now need to prove that the GK-dimension of $\operatorname{Ind}_{D}^{H} M(\Lambda)$ cannot be larger than $\operatorname{dim} G / P_{\Lambda}$. Let $L=\mathbb{Z} \Lambda$. By the lemma above, $L$ has a $\mathbb{Z}$-basis $\left\{\lambda_{1}, \ldots, \lambda_{r}\right\}$ such that $\Lambda \subseteq \sum_{i=1}^{r} \mathbb{N} \lambda_{i}$. For each $n$, we define

$$
\Lambda_{n}=\left\{\lambda \in \Lambda \mid \lambda=\sum_{i=1}^{r} n_{i} \lambda_{i}, n_{i} \in \mathbb{N}, \sum_{i=1}^{r} n_{i}=n\right\} .
$$

Note that all $n_{i} \in \mathbb{N}$ and $\Lambda_{n}+\Lambda_{m} \subseteq \Lambda_{n+m}$. This defines a graded algebra structure on $\operatorname{Ind}_{D}^{H}(M(\Lambda))=\oplus_{n=0}^{\infty} \operatorname{Ind}_{D}^{H}\left(M\left(\Lambda_{n}\right)\right)$.

Using the Weyl dimension formula, we have

$$
\begin{aligned}
\operatorname{dim} \operatorname{Ind}_{D}^{H} M\left(\Lambda_{n}\right) & =\sum_{\lambda \in \Lambda_{n}} \operatorname{dim}(\lambda) \\
& \leq \sum_{\left(n_{1}, \cdots, n_{r}\right) \in C(r, n)} \prod_{j=1}^{N}\left|1+\sum_{i=1}^{r} n_{i} a_{i j}\right| \\
& \leq \sum_{\left(n_{1}, \cdots, n_{r}\right) \in C(r, n)} \prod_{j=1}^{N}\left(1+\sum_{i=1}^{r} n_{i}\left|a_{i j}\right|\right)=d(n),
\end{aligned}
$$

where $a_{i j}=\left\langle\lambda_{i}, \alpha_{j}^{\vee}\right\rangle$. Proposition 3.11, $d(n)$ is a polynomial of degree $N+r-1-\left|J_{L}\right|$. Then the sequence $f(n)=\operatorname{dim}\left(\oplus_{m=0}^{n} \operatorname{Ind}_{D}^{H} M\left(\Lambda_{m}\right)\right)$ has growth rate not larger than $N+r-\left|J_{L}\right|$.

Let $A$ be a finitely generated subalgebra of $\operatorname{Ind}_{D}^{H}(M(\Lambda))$. There exist $n_{0}$ such that the generators are in $V=\oplus_{m=0}^{n_{0}} \operatorname{Ind}_{D}^{H} M\left(\Lambda_{m}\right)$. We can assume that $A$ is generated by $V$, then $V^{n} \subseteq \oplus_{m=0}^{n n_{0}} \operatorname{Ind}_{D}^{H} M\left(\Lambda_{m}\right)$. Thus $\operatorname{dim} V^{n} \leq f\left(n n_{0}\right)$. This shows that the GK-dimension of $A$ is at most $N+r-\left|J_{\Lambda}\right|$. Therefore, by the definition, the GK-dimension of $\operatorname{Ind}_{D}^{H} M(\Lambda)$ is at most $N+r-\left|J_{\Lambda}\right|$.

Since $N-\left|J_{\Lambda}\right|=\operatorname{dim} G / P_{\Lambda}$, we thus have $G K-\operatorname{dim}\left(\operatorname{Ind}_{D}^{H} M(\Lambda)\right)=\operatorname{dim} G / P_{\Lambda}+$ $\operatorname{rank} \mathbb{Z} \Lambda$.

Remark 1. If $H=\operatorname{Dist} G$ and $D=\operatorname{Dist}(B)$ for a reductive group $G$ with a Borel subgroup $B$, then the GK-dimension of $\operatorname{Ind}_{D}^{H} M(\Lambda)$ is the same as the Krull dimension and the dimension of the quotient variety $G / U$ with $U$ being the unipotent radical of $B$. However for the quantum groups, this algebras are not commutative. 
3.18 Note that the algebra $M(\Lambda)$ is graded when a basis as in the above proof for $L=\mathbb{Z} \Lambda$ is given. $\operatorname{dim} M\left(\Lambda_{n}\right)=\left(\begin{array}{c}r+n-1 \\ r-1\end{array}\right)$ which is a polynomial of $n$ of degree $r-1$. The above proof also shows that $\operatorname{GK}-\operatorname{dim}(M(\Lambda))=\operatorname{rank}(\mathbb{Z} \Lambda)$ for any submonoid $\Lambda$ of $X_{+}$.

Thus the above results suggest that $\mathrm{GK}-\operatorname{dim} \operatorname{Ind}_{D}^{H}(M) \leq \operatorname{dim} G / P+\operatorname{GK}-\operatorname{dim}(M)$ for any $D$-module algebra $M$, where $P$ is parabolic subgroups of $G$ such that $M$ extends to the a $P$-module structure (or its quantum analog).

\section{Final REMARKS}

4.1 Let $H$ be the quantum enveloping algebra over $k=\mathbb{C}(q)$. The canonical basis defined by Lusztig defines a canonical basis for each $\operatorname{Ind}_{D}^{H} k_{\lambda}$ through the dual basis of the canonical basis of the quantum Weyl module for all $\lambda \in X_{+}$. The $\mathbb{Z}\left[q, q^{-1}\right]$-lattice $A_{\mathbb{Z}\left[q, q^{-1}\right]}(\lambda)$ generated by this basis in $\operatorname{Ind}_{D}^{H} k_{\lambda}$ respects the tensor product in the sense that the multiplication $\operatorname{Ind}_{D}^{H} k_{\lambda} \otimes \operatorname{Ind}_{D}^{H} k_{\mu} \rightarrow \operatorname{Ind}_{D}^{H} k_{\mu+\lambda}$ sends $A_{\mathbb{Z}\left[q, q^{-1}\right]}(\lambda) \otimes_{\mathbb{Z}\left[q, q^{-1}\right]} A_{\mathbb{Z}\left[q, q^{-1}\right]}(\mu)$ to $A_{\mathbb{Z}\left[q, q^{-1}\right]}(\lambda+\mu)$ (see the [L1, 25.1.2]). One can also define the induced modules by taking $\mathbb{Z}\left[q, q^{-1}\right]$ as the ground ring instead of the field $k$. Let $H_{\mathbb{Z}\left[q, q^{-1}\right]}$ and $D_{\mathbb{Z}\left[q, q^{-1}\right]}$ be the $\mathbb{Z}\left[q, q^{-1}\right]$-form in $H$ and $D$ respectively, then $A_{\mathbb{Z}\left[q, q^{-1}\right]}(\lambda)=\operatorname{Ind}_{D_{\mathbb{Z}\left[q, q^{-1}\right]}}^{H_{\mathbb{Z}\left[q, q^{-1}\right.}} \mathbb{Z}\left[q, q^{-1}\right]_{\lambda}$. In this way we can get a $\mathbb{Z}\left[q, q^{-1}\right]$-form $A_{\mathbb{Z}\left[q, q^{-1}\right]}(\Lambda)=\oplus_{\lambda \in \Lambda} A_{\mathbb{Z}\left[q, q^{-1}\right]}(\lambda) \operatorname{in} \operatorname{Ind}_{D}^{H} M(\Lambda)$ for each submonoid $\Lambda$ see $[\mathrm{APW}]$ and [Lin3].

4.2 For any field $k$ and any ring homomorphism $\mathbb{Z}\left[q, q^{-1}\right] \rightarrow k$, we can form the $k$-Hopf algebras $H_{k}=k \otimes_{\mathbb{Z}\left[q, q^{-1}\right]} H_{\mathbb{Z}\left[q, q^{-1}\right]}$ and $D_{k}=k \otimes_{\mathbb{Z}\left[q, q^{-1}\right]} D_{\mathbb{Z}\left[q, q^{-1}\right]}$. Then define $\operatorname{Ind}_{D_{k}}^{H_{k}} M_{k}(\Lambda)$. An easy computation will show that $\operatorname{Ind}_{D_{k}}^{H_{k}} M_{k}(\Lambda) \cong k \otimes_{\mathbb{Z}\left[q, q^{-1}\right]}$ $A_{\mathbb{Z}\left[q, q^{-1}\right]}(\Lambda)$. In particular, if $\mathbb{Z}\left[q, q^{-1}\right] \rightarrow k$ sends $q$ to 1 , then $\operatorname{Ind}_{D_{k}}^{H_{k}} M_{k}(\Lambda)$ is the algebra we constructed for the corresponding semisimple algebraic group $G$. Thus $\operatorname{Ind}_{D}^{H} M(\Lambda)$ is a quantum deformation of the corresponding algebra of the semisimple algebra group.

4.3 It is also interesting to note that for the distribution algebra of semisimple algebraic groups in positive characteristic $p$, the GK-dimension is 0 since the algebra itself is a direct union of finite dimensional hyperalgebras. This shows that different $\mathbb{Z}$ forms in the enveloping algebra of the complex semisimple Lie algebra could give rise to $k$-algebras with different GK-dimensions. However, for the quantum enveloping algebras defined by Lusztig using "divided" powers, then GK-dimension does not change when the base field is of characteristic 0 even in the root of unity cases..

4.4 For fixed and algebraic group $G$ and Borel subgroup $B$, the algebra $\operatorname{Ind}_{B}^{G} M(\Lambda)$ is a subalgebra of the coordinate algebra $k[G]$. In fact, $\operatorname{Ind}_{D_{k}}^{H_{k}} M_{k}(\Lambda)=\oplus_{\mu \in-\Lambda} k[G]_{\mu}$ 
is the subalgebra of $B$-semi-invariants in $k[G]$. Here $G$ acts on $k[G]$ from left by $(b f)(x)=f\left(b^{-1} x\right)$ for all $x \in G$ and all $b \in G$. Since $G$ is connected, this algebra is an integral domain. There is a natural gradation defined by the height of weights in $X_{+}$in the sense of $\operatorname{ht}(\lambda)=\sum_{i=1}^{l} n_{i}$ if $\lambda=\sum_{i=1}^{l} n_{i} \omega_{i}$, with $\omega_{1}, \cdots, \omega_{l}$ be the fundamental weights. This graded algebra defines an irreducible projective scheme $X(\Lambda)$, on which $G$ acts. The flag variety $G / B$ is the special case. If $\Lambda=X_{+}$, then $A(\Lambda)$ contains the homogeneous coordinate algebra of $G / B$ as a subalgebra. This defines a surjective $G$-equi-variant map $X(\Lambda) \rightarrow G / B$. An interesting question is to describe the geometry of the projective scheme $X(\Lambda)$, and their relation with the representations of the algebraic groups, in particular, the cohomology groups of line bundles of over the flag varieties.

4.5 For finite dimensional algebra $A$ and a finite dimensional $A$-module $M$, let $\cdots \rightarrow P_{1} \rightarrow P_{0} \rightarrow M \rightarrow 0$ be the minimal projective resolution. Define $f(n)=\operatorname{dim} P_{n}$ for all $n \in \mathbb{N}$. The complexity of $M, c(M)$ is defined to be $\nu(f)+1$.

On the other hand, $\operatorname{Ext}_{A}^{*}(M, M)=\oplus_{n=0}^{\infty} \operatorname{Ext}_{A}^{n}(M, M)$ is a graded $k$-algebra with

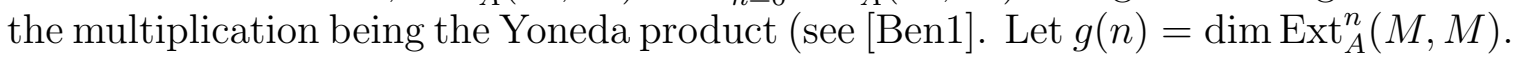
It is easy to see that $\mathrm{GK}-\operatorname{dim}\left(\operatorname{Ext}_{A}^{*}(M, M)\right) \leq \nu(g)+1$. One the other hand, following an argument as in [Ben2, 5.3] we always have $\nu(g) \leq c(M)-1$. If $\operatorname{Ext}_{A}^{*}(M, M)$ is finitely generated as a $k$-algebra, then $\operatorname{GK}-\operatorname{dim}\left(\operatorname{Ext}_{A}^{*}(M, M)\right)=\nu(g)+1$. Furthermore, if $\operatorname{Ext}_{A}^{*}(M, M)$ is finitely generated as a $k$-algebra and $\operatorname{Ext}_{A}^{*}(M, S)$ is a finitely generated $\operatorname{Ext}_{A}^{*}(M, M)$-module, then we have $\operatorname{GK}-\operatorname{dim}\left(\operatorname{Ext}_{A}^{*}(M, M)\right)=c(M)$. Note that the complexity $c(M)$ was defined as an integer in [Ben2]. But the definition we give here is more natural.

Friedlander and Suslin [FS] have provedthat for a finite algebraic group scheme $G$ and any finite dimensional rational $G$-module $M, \operatorname{Ext}_{A}^{*}(M, M)$ is finitely generated as a $k$-algebra and $\operatorname{Ext}_{A}^{*}(M, N)$ is a finitely generated as $\operatorname{Ext}_{A}^{*}(M, M)$-module. Note that in this case the Yoneda product coincides with the cup product, and $\operatorname{Ext}_{A}^{*}(M, M)$ is graded commutative. Thus the Hilbert series of the graded vector space $\operatorname{Ext}_{A}^{*}(M, N)$ has the form $p(t) / \prod\left(1-t_{i}^{n}\right)$ with $p(t)$ being a polynomial. There-

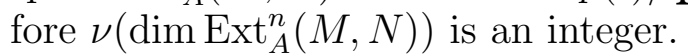

Now for any finite dimensional Hopf algebra $H$, the category of all $H$-modules is isomorphic to the category of all $H^{\circ}$-comodules. Here $H^{\circ}$ is a finite dimensional Hopf algebra. (See [Lin1]). Thus we have

Theorem 4.6. If $A$ is a finite dimensional cocommutative Hopf algebra, then for any finite dimensional $H$-module $M$, we have $\mathrm{GK}-\operatorname{dim}(M)=c(M)$ which is always an integer.

\section{REFERENCES}

[APW] H.H. Andersen, P. Polo, K. Wen, Representations of quantum algebras, Invent. Math. 104 (1991), 1-59. 
[ATV] M. Artin, J. Tate and M. Van der Bergh, Modules over regular algebras of dimension 3, Invent. Math. 106 (1991), 335-388.

[Ben1] D.J. Benson, Representations and cohomology I: Basic representations theory of finite groups and associative algebras, Cambridge University Press, 1991.

[Ben2] D.J. Benson, Representations and cohomology II: Cohomology of groups and modules, Cambridge University Press, 1991.

[C] J. Carlson, Complexity and Krull dimension. in Representations of algebras (Puebla, 1980), pp. 62-67, Lecture Notes in Math., 903, Springer, Berlin/New York, 1981.

[Don] S. Donkin, Rational representations of algebraic groups: tensor products and filtrations, (Lecture Notes in Mathematics 1140), Springer-Verlag, Berlin/Heidelberg/New York/Tokyo 1985.

[FS] E. Friedlander, A. Suslin, Cohomology of finite group schemes over a field. Invent. Math. 127 (1997), no. 2, 209-270.

[Ha] R. Hartshorne, Algebraic Geometry, Springer-Verlag, New York, 1977.

[Hu3] J. E. Humphreys, Introduction to Lie Algebras and Representation Theory, Gradate Text Book in Mathematics 9, Springer-Verlag, New York/Heidelberg/Berlin 6th printing, 1994.

[Jan] J.C. Jantzen, Representations of Algebraic Groups, Academic Press, 1987.

[KL] G. Krause and T. H. Lenagan, Growth of algebras and Gelfand-Kirillov dimension, Research Notes in Mathematics, Pitman Adv. Publ. Program, vol 116 (1985).

[Lin1] Z. Lin, Induced representations of Hopf algebras: applications to quantum groups at roots of 1, J. Algebra 154 (1993), 152-187.

[Lin2] Z. Lin, A Mackey decomposition theorem and cohomology for the quantum groups at roots of 1, J. Algebra 166 (1) (1994), 100-129.

[Lin3] Z. Lin, Freeness of quantum coordinate algebra, (unpublished).

[LN1] Z. Lin, D.K. Nakano, Algebraic group actions in the representation and cohomology theory of Lie algebras of Cartan type, to appear in J. Algebra.

[LMO] A. Leroy, J. Matczuk and J. Okninski, On the Gelfand-Kirillov dimension of normal localizations and twisted polynomial rings, Perspectives in Ring Theory, F. van Oystaeyen and L. Le Bruyn, Editors, Kluwer Academic Publishers (1988), 205-214.

[L1] G. Lusztig, Introduction to Quantum Groups, Birkhäuser, Boston/Basel/Berlin 1994

[L2] G. Lusztig, Canonical basis arising from quantized enveloping algebras II, (pp. 175-201) in Common Trends in Mathematics and Quantum Field Theories, (T. Eguchi et. al., eds.) Progr. Theor. Phys. Supll., Vol. 102, 1990

[L3] G. Lusztig, Quantum groups at roots of 1, Geom. Dedicata 35 (1990), 89-113.

[M] O. Mathieu, Filtrations of G-modules, Ann. Sci. École Norm. Sup. 23 (1990), 625-644.

[MR] J. C. McConnell and J. C. Robson, Non-Commutative Noetherian Rings, Wiley-interscience, Chichester, 1987.

[Mu I. Musson, Gelfand-Kirillov dimension of twisted Laurent Extensions, Comm. Alg. 17 (11), 1989, 2853-2856.

[O] C. Ohn, "Classical" flag varieties for quantum groups: the standard quantum $S L(n, \mathbb{C})$, Preprint (http://arXiv.org/abs/math.AG/0007005).

[P] J. Paradowski, Filtrations of modules over the quantum algebra, Proceedings of Symp. Pure Math. Ed. Haboush and Parshall 56 Part 2 (1994), 93-108.

[Sw] M. Sweedler, Hopf Algebras, Benjamin, New York, 1974.

[W] Jian-pan Wang, Sheaf cohomology on $G / B$ and tensor products of Weyl modules, J. Algebra 77 (1982), 162-185

[Zh1] J. Zhang, On Gelfand-Kirillov transcendence degree, Trans. Amer. Math. Soc. 348 (1996), no. $7,2867-2899$. 
[Zh2] J. Zhang, A note on GK-dimension of skew polynomial extensions, Proc. Amer. Math. Soc. 125 (1997), no. 2, 363-373.

Department of Mathematics, Kansas State University, Manhattan, KS 66506

E-mail address: zlin@math.ksu.edu 